\title{
INVESTIGACIONES
}

\section{La cartografía social como estrategia didáctica: reconociendo recorridos e imaginarios}

\author{
Social cartography as a didactic strategy: \\ recognizing routes and imaginaries \\ A cartografia social como estratégia didática: \\ reconhecendo caminhos e imaginários \\ Diana Yaneth Ávila Camargo $^{a}$ \\ ${ }^{a}$ Universidad Politécnica Salesiana, sede Quito, Ecuador. \\ davilac@ups.edu.ec
}

\begin{abstract}
RESUMEN
Como parte de la asignatura de Didáctica de Ciencias Sociales de la carrera de Licenciatura en Educación Intercultural Bilingüe de la Universidad Politécnica Salesiana, sede Quito-Ecuador, se presenta la necesidad de aportar recursos didácticos que permitan a los estudiantes un reconocimiento de sus territorios, así como de estrategias didácticas y metodológicas para la enseñanza de la geografía y que se utilicen como aporte para la preservación de la memoria colectiva de sus comunidades. De esta manera se propone en el Grupo de Investigación de Educación e Interculturalidad de la misma universidad la realización del proyecto de investigación "La cartografía social como estrategia didáctica, reconociendo recorridos e imaginarios con los estudiantes de Licenciatura en Educación Intercultural Bilingüe de la Universidad Politécnica Salesiana", dicha investigación tuvo como punto de partida el reconocimiento previo del contexto geográfico en el que se encuentran los estudiantes y la implementación de la cartografía social como estrategia didáctica para la enseñanza de la geografía. Metodológicamente se ha utilizado la Investigación Acción Participativa (IAP), y la aplicación de la cartografía social como estrategia pedagógica y didáctica para la enseñanza de las ciencias sociales y cómo motivación para el desarrollo de estrategias que les permitan acercarse a los futuros docentes a sus estudiantes, comprendiendo sus contextos y realidades, partiendo del reconocimiento y representación del espacio en que viven; y asimismo sea una oportunidad de intercambio de saberes entre estudiantes y docentes.
\end{abstract}

Palabras claves: Cartografía Social, Ciencias Sociales, Estrategias didácticas, Educación Intercultural Bilingüe, Geografía, Investigación Acción Participativa.

\begin{abstract}
As part of the Social Sciences Didactics course of the Bachelor's degree in Intercultural Bilingual Education at the Salesian Polytechnic University, Quito-Ecuador, the need to provide didactic resources that allow students to recognize their territories is presented, as well how of didactic and methodological strategies for the teaching of geography and that they are used as a contribution to the preservation of the collective memory of their communities. In this way, the Research Group on Education and Interculturality of the same university is proposed to carry out the research project "Social cartography as a didactic strategy, recognizing routes and imaginations with the Bachelor's degree students in Intercultural Bilingual Education of the Salesian Polytechnic University", this research had as its starting point the prior recognition of the geographical context in which the students find themselves and the implementation of social cartography as a didactic strategy for teaching geography. Methodologically, Participatory Action Research (IAP) has been used, and the application of social cartography as a pedagogical and didactic strategy for teaching social sciences and as motivation for the development of
\end{abstract}

Recibido: 2020/09/04

Aceptado: 2020/10/26 
strategies that allow future teachers to approach their students, understanding their contexts and realities, starting from the recognition and representation of the space in which they live; and also be an opportunity to exchange knowledge between students and teachers.

Key words: Social Cartography, Social Sciences, Didactic Strategies, Intercultural Bilingual Education, Geography, Participatory Action Research.

\section{RESUMO}

No âmbito do curso de Didática das Ciências Sociais do Bacharelado em Educação Intercultural Bilíngue da Universidade Politécnica Salesiana, sede de Quito-Equador, apresenta-se também a necessidade de disponibilizar recursos didáticos que permitam aos alunos reconhecer seus territórios como de estratégias didáticas e metodológicas para o ensino de geografia e que são utilizadas como contribuição para a preservação da memória coletiva de suas comunidades. Desta forma, propõe-se no Grupo de Investigação em Educação e Interculturalidade da mesma universidade a realização do projecto de investigação "Cartografia social como estratégia didáctica, reconhecendo percursos e imaginários com os alunos do Bacharelado em Educação Intercultural Bilíngue da Universidade Politécnica Salesiana", Dita pesquisa teve como ponto de partida o reconhecimento prévio do contexto geográfico em que os alunos se inserem e a implementação da cartografia social como estratégia didática para o ensino da geografia. Metodologicamente, tem-se utilizado a Pesquisa-Ação Participativa (PAR), e a aplicação da cartografia social como estratégia pedagógica e didática para o ensino das ciências sociais e como motivação para o desenvolvimento de estratégias que permitam aos futuros professores se aproximarem de seus alunos. , compreendendo seus contextos e realidades, a partir do reconhecimento e representação do espaço em que vivem; e também ser uma oportunidade de troca de conhecimentos entre alunos e professores.

Palavras-chave: Cartografia Social, Ciências Sociais, Estratégias de Ensino, Educação Intercultural Bilíngue, Geografia, Pesquisa Ação Participativa.

\section{INTRODUCCIÓN}

En Ecuador la Educación Intercultural Bilingüe (EIB) se constituye en un importante logro del movimiento indígena y de las lideresas indígenas que impulsaron el proceso en el siglo $\mathrm{XX}$, hablamos de Dolores Cacuango quien expondría la necesidad de alfabetización del pueblo indígena en los años 40, con la creación de las llamadas "Escuelas Clandestinas de Cayambe" y Tránsito Amaguaña quien seguiría apoyando el proceso de la mano del movimiento indígena hasta el reconocimiento de la EIB en 1988, que daría paso a la creación de la DINEIB (Dirección Nacional de Educación Intercultural Bilingüe) y la necesidad de construir un currículo propio llamado MOSEIB (Modelo del Sistema de Educación Intercultural Bilingüe), así como la elaboración de recursos didácticos propios, teniendo en cuenta las diferentes lenguas y contextos de los pueblos y nacionalidades indígenas del Ecuador.

En este marco surge el Proyecto Académico Cotopaxi (PAC), el cual "constituye un programa de licenciatura en Educación Intercultural Bilingüe, fue creado para profesionalizar a los docentes indígenas de las zonas rurales de la provincia de Cotopaxi" (Granda Merchán e Iza Remache, 2012, p. 662). Lo anterior como necesidad a partir del crecimiento del Sistema de Escuelas Interculturales de Cotopaxi (SEIC), motivado por "los dirigentes indígenas de la zona conjuntamente con la Pastoral de Zumbahua (integrada por la comunidad salesiana y la organización italiana de voluntarios Matto Grosso)" (Granda Merchán e Iza Remache, 2012, p. 664) sistema que data el origen de la primera escuela en 1976. El PAC se adscribe como parte de los programas ofertados por la naciente Universidad Politécnica Salesiana (UPS) en 1994. 
Así, el PAC crece hasta consolidarse como la carrera de Licenciatura en Educación Intercultural Bilingüe y abrir su cobertura en otras provincias del territorio ecuatoriano, en los llamados "Puntos focales" hoy llamados "Centro de Apoyo", que serían en orden de aparición: Latacunga (2003), "Otavalo - provincia de Imbabura (2003), punto focal Cayambe - provincia de Pichincha (2004), punto focal Wasak'entza - provincia de Morona Santiago (2005), punto focal Simiatug - provincia de Bolívar (2006), y punto focal Riobamba provincia de Chimborazo (2008) (Granda Merchán e Iza Remache, 2012, p. 667).

La presente ponencia se enfoca en el trabajo de investigación titulado "La cartografía social como estrategia didáctica, reconociendo recorridos e imaginarios con los estudiantes de Licenciatura en Educación Intercultural Bilingüe de la Universidad Politécnica Salesiana" realizado con la participación de los estudiantes de los centros de apoyo de Otavalo, Cayambe, Latacunga, Riobamba, Simiatug en la sierra Norte y central de Ecuador, y Wasakentsa, misión salesiana ubicada en el oriente ecuatoriano o región Amazónica, como parte de las actividades de los encuentros presenciales de la asignatura de didáctica de las ciencias sociales de tercer semestre.

El objetivo principal de la investigación es reconocer la cartografía social como herramienta didáctica y metodológica para el ejercicio del docente intercultural bilingüe, la que permite el reconocimiento de territorios y espacios ancestrales como parte del proceso de construcción de memoria colectiva. Lo anterior a partir del reconocimiento de la importancia y compromiso social del docente intercultural bilingüe, teniendo en cuenta la rápida contextualización histórica presentada al inicio del documento; así como el reconocimiento de sus lugares de origen y sus posibles lugares de trabajo, es decir, con espacios propios que hacen parte de la memoria colectiva de las comunidades que habitan, por tanto el proyecto es de gran relevancia para la revaloración de sus espacios y territorios, a partir del uso de la cartografía social como estrategia que permite expresar por medio de símbolos e imágenes, variada y valiosa información que los estudiantes guardan en sus historias de vida y como actores comunitarios.

Como punto de partida realizaremos un acercamiento a la metodología aplicada en la investigación, especificando su uso pedagógico y didáctico, y su implementación como estrategia en el espacio académico para la enseñanza de las ciencias sociales. Así posibilitar en los futuros docentes la creación de estrategias otras que les permitan acercarse a sus estudiantes, comprendiendo sus contextos y realidades, partiendo del reconocimiento y representación del espacio en que viven, ya sea empleando herramientas de la geografía y la cartografía presentes en los planes de estudio y/o como opción de intercambio de saberes entre docentes.

Posteriormente nos encontramos con los resultados principales de la investigación luego de la realización de la actividad propuesta en la metodología, retomando algunos ejemplos de los trabajos elaborados por los estudiantes y la discusión retomando algunos conceptos y categorías, a partir de la revisión del aporte de autores y experiencias investigativas que han trabajado con la cartografía social en comunidades y/o en espacios educativos diversos; finalmente se presentarán algunas conclusiones o reflexiones finales.

\section{METODOLOGÍA}

La investigación se realizó con la participación de los estudiantes de la carrera de Licenciatura en Educación Intercultural Bilingüe (EIB) de la Universidad Politécnica Salesiana (UPS), 
en los espacios de encuentros presenciales en los diferentes centros de apoyo durante el curso de su tercer semestre, donde se encontraba dentro de la malla curricular la asignatura de Didáctica de las Ciencias Sociales. Para poder realizar la experiencia con todos los grupos fue necesario pasar algunos semestres para completar la información y así poder realizar la rotación docente en todos los centros de apoyo, finalmente se obtiene el material requerido para el análisis y presentación de resultados de la investigación. En los encuentros presenciales se organizaba el grupo general en mesa redonda y se les proponía la actividad partiendo de la siguiente pregunta: ¿Cómo es el recorrido que realizo de mi casa al centro de apoyo?, lo anterior retomando los aportes de Paulo Freire y la Pedagogía de la Pregunta (Freire y Faundez, 2013).

La respuesta debía plasmarse gráficamente en un plano o croquis realizado en una hoja, para esto debían especificar el recorrido desde sus hogares y los principales elementos que encontraban alrededor, allí se incluían lugares específicos, sean geográficos (montañas, ríos, lagunas, valles, etc.) o de tránsito (tiendas, escuelas y colegios, iglesias, plazas de mercado, instituciones públicas, hospitales, etc.). Estos lugares debían representarlos empleando signos convencionales creados por ellos, especificando el tiempo de duración del recorrido, y cómo lo realizaba (caminando, y/o utilizando medios de transporte), especificando cuáles y asimismo representándolos (bus interprovincial, bus intercantonal, bus urbano, carro particular, motocicleta, bicicleta, canoa, etc.). Por último, debían indicar con flechas o guías de color el recorrido y dibujando la rosa de los vientos como herramienta básica de la cartografía, para definir su ubicación en los puntos cardinales.

Es importante tener presente que antes de la actividad, en la mayoría de los casos se tuvo el conocimiento de los lugares o comunidades de origen de los estudiantes, o sus alrededores, a partir de la experiencia previa de trabajo en la carrera; y también por los desplazamientos propios de los docentes para asistir a los encuentros presenciales en los centros de apoyo mencionados. También, se tuvo en cuenta la realización de viajes particulares por las zonas de afluencia de los estudiantes que forman parte de la población objetiva que ingresa a la carrera.

Teniendo claras las indicaciones presentadas al inicio del taller, los estudiantes realizaron de forma individual, pero siempre comentando en intercambio con sus compañeros, el plano del recorrido de sus hogares al centro de apoyo. Describiendo gráficamente lo que encontraban a su paso e indicando la orientación del recorrido a partir del uso de los puntos cardinales, destacando espacios de encuentro, y lugares importantes como montañas, ríos, comunidades, parroquias, cantones y ciudades, centros de salud, escuelas y colegios, entre otros.

El acompañamiento y guía en el proceso de elaboración de los planos de recorrido fue importante, ya que en el momento de realización de la actividad se apoyaba su elaboración planteándoles más preguntas según cada caso, por ejemplo, ¿Qué montañas o ríos hay alrededor? ¿Cuánto tiempo me demoro en el desplazamiento? ¿Qué lugares son los más relevantes?, etc., se solicitaban diversas indicaciones "que me permitan llegar a sus casas y no perderme en el camino"1.

De esta manera se motivaba a los estudiantes para la elaboración de sus planos de recorridos, también la simbología creada y representada, a partir de las percepciones

Frases como esta fueron empleadas para motivar el trabajo de los estudiantes y que concluyeran la actividad en el tiempo previsto. 
propias y referencias sobre el uso de signos convencionales básicos. Estos símbolos y signos eran reproducidos o creados para especificar cruces, puentes, líneas férreas, ríos, hoteles, restaurantes, autopistas, cambios de caminos, instituciones educativas, hospitales, iglesias, entidades gubernamentales, etc.

Así, los estudiantes reconocían a partir de la elaboración y representación de sus recorridos, lugares comunes por medio de un ejercicio de memoria, recuperando recuerdos, imágenes de los sitios transitados cotidianamente, pero de los cuales muchas veces no prestaban mayor atención, así como de las personas y otros espacios que se cruzaban en su recorrido, grandes montañas (como los volcanes Cayambe, Chimborazo, Tungurahua, Imbabura, Cotacachi, entre otros), ríos, lagunas, lagos, valles, etc.; que forman la geografía que los estudiantes recorren en su ir y venir, desde las comunidades a los centros urbanos. Además, lugares de encuentro sean parques centrales, paradas de bus, terminales de transporte, plazas de mercado, iglesias, y/o casas de familiares y amigos, lugares o espacios como referencias claves de su recorrido.

Al finalizar la actividad se realizó la socialización de algunos de los trabajos, y allí los compañeros encontraban similitudes y diferencias entre los planos, también llamaba la atención las formas de representación y símbolos empleados y creados, así como los tiempos entre las distancias recorridas, y los medios empleados para su desplazamiento, también se presentaron casos en los que se encontraban cerca del centro de apoyo y otros en los que las distancias y condiciones para llegar al mismo era considerables. Lo anterior permitió una mayor comprensión y valoración por parte de los compañeros del curso sobre los esfuerzos que realizan en su formación universitaria.

\section{RESULTADOS}

Entre los principales resultados de la investigación se encuentra la identificación de los lugares de procedencia de los estudiantes, fue de gran relevancia tanto para los estudiantes como para la docente conocer gráficamente los lugares de vivienda y lo que se encuentra en su recorrido, así se reconocieron lugares claves en los recorridos, que para ellos en un principio eran normales o pasaban desapercibidos sean estos naturales o artificiales.

Fue importante también el reconocimiento de las distancias recorridas y los tiempos empleados en el desplazamiento de los estudiantes para llegar a los encuentros presenciales de la carrera. Como se mencionaba en el apartado final de la metodología, permite una comprensión de los esfuerzos que los estudiantes realizan para asistir a los encuentros presenciales, así como poder ser parte de eventos en la sede Quito (Inti Raimi, Congresos, Ceremonias de grados, entre otros) y demás situaciones propias de la vida universitaria (trámites administrativos, etc.).

La elaboración del plano permitió la identificación de habilidades y competencias de ubicación espacial y uso de elementos propios de la cartografía, algunos realizaban las representaciones a partir de referencias previas. Vale comentar que antes del taller se realiza con los estudiantes la presentación de elementos básicos de la representación cartográfica y algunos ejemplos de estos, tipos de mapas, planos, signos convencionales, rosa de los vientos y espacios comunes donde se pueden encontrar, por ejemplo, en los espacios escolares, así como en lugares turísticos, en trámites de compra-venta de terrenos, uso de aplicaciones tecnológicas, etc. 
Los estudiantes reconocieron en la cartografía una estrategia didáctica para el trabajo en aula, muchos de ellos no tienen buenos recuerdos de las clases de ciencias sociales, por lo que la asocian con temas de memorización y repetición de fechas, lugares y personajes. Por ejemplo, la geografía se torna como algo muy lejano, aunque tengan referentes importantes en sus territorios (como se comentó anteriormente en la representación y ubicación de volcanes, ríos, lagunas y lagos significativos en cada zona).

Al hacer el ejercicio de memoria y reconocimiento del espacio habitado, los estudiantes recordaron además de los espacios geográficos, relatos de sus mayores, de sus familias, de las personas de la comunidad en general sobre la zona que habitan, lo que permite generar procesos de recuperación de la historia oral, a partir del recordar y compartir historias de la zona, de las relaciones entre montañas, del nacimiento de los ríos, de las entidades protectoras de las lagunas, entre otros.

Reconocieron además en sus planos de recorrido algunas habilidades o destrezas en ellos mismos, que, como dato importante en varios de los casos analizados en la observación posterior de los planos, eran muy destacables los trabajos realizados por estudiantes que tiene falencias en la lectura y/o escritura, y que al realizar un trabajo gráfico tuvieron mayor facilidad de dar a conocer sus conocimientos y expresarlos a partir de los dibujos y símbolos creados. Lo anterior se evidencia por ejemplo en el uso del color, el alto nivel de detalle en sus dibujos, el compromiso de realizar muy bien su plano para que la docente pueda llegar a sus hogares, entre otros elementos que llenan de mucho valor sus trabajos y son reflejo claro de la importancia de aplicar otras formas de aprendizaje y evaluación, lo cual fue reconocido por los estudiantes para tomar en cuenta en sus espacios de trabajo docente.

Finalmente, se destaca como resultado de la investigación la validez del empleo de la cartografía social como estrategia didáctica para aplicar con estudiantes jóvenes-adultos universitarios que sin importar sus antecedentes de formación primaria y secundaria, guardan habilidades particulares que han desarrollado en sus espacios educativos u otros espacios de aprendizaje, retomando la importancia de la construcción de mapas colectivos dentro de la Investigación Acción Participativa (IAP), pero llevándolo al espacio escolar para que ellos la reconozcan como estrategia didáctica importante para acercar a sus estudiantes al aprendizaje de la geografía, desde el reconocimiento de sus contextos, más allá de procesos de memorización y repetición, y así ir poco a poco ampliando la escala (De Sousa Santos, 1991).

\section{DISCUSIÓN}

Partimos de entender la cartografía social como "un método de construcción de mapas -que intenta ser- colectivo, horizontal y participativo" (Tetamanti, 2014, p. 15). De esta manera se trabaja como metodología retomando los aportes de Paulo Freire para la Educación Popular (Freire y Faundez, 2013) y Orlando Fals Borda para la Investigación Acción Participativa (IAP) (Fals Borda, 2017).

Cada uno de los autores mencionados trabaja desde sus investigaciones la importancia del reconocimiento del espacio por parte de las personas que habitan un territorio, allí entra en juego la relevancia del conocimiento, por ejemplo, de elementos básicos en la enseñanza de las ciencias sociales como la ubicación espacial y geográfica, para el reconocimiento y 
ubicación en los territorios y la comprensión de las relaciones socio-espaciales que se tejen en estos. Lo anterior teniendo en cuenta que:

El territorio, (...), puede ser entendido como una construcción social que se desarrolla a partir de las significaciones y usos que los sujetos construyen cotidianamente, a partir de historias comunes, usos y sentidos. Así como sujetos somos seres con historia, el territorio también la tiene y esa historicidad es construida en forma colectiva. (...) En el territorio es el lugar donde la identidad y la pertenencia son constituidas como fundamentos de la cohesión social, ya que éste es habitado por la memoria y la experiencia. (Carballeda, 2014, pp. 26-27).

Figura 1. Fotografía propia - Centro de apoyo Otavalo

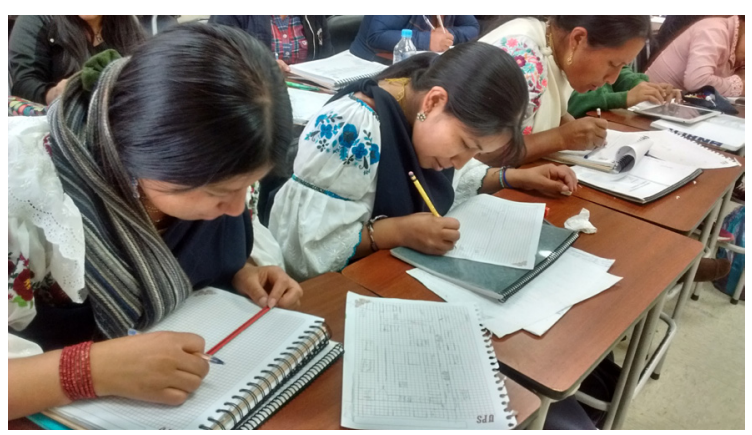

Fuente: Elaboración propia.

Sin olvidar que la cartografía social:

Asume el mapa como una representación convencional gráfica de fenómenos concretos o abstractos, localizados en un contexto determinado. Dado que el mapa es un esquema de la realidad, constituido de signos, símbolos y/o palabras, su construcción debe obedecer a acuerdos y prácticas colectivas, conducentes a la producción de convenciones, las cuales hacen posible la producción de significados compartidos y proyecciones colectivas. (Barragán y Amador, 2014, p. 134).

De igual manera las categorías de representación y orientación son importantes ya que los planos elaborados por los estudiantes privilegian la orientación más allá de la representación, aunque la representación de sus espacios es fundamental dentro de ese proceso, a partir del uso de signos convencionales, representación de lugares específicos. Ya que:

La representación expresa el significado de las cosas, los lugares, las personas, los colectivos, las organizaciones, las autoridades; el entorno natural y construido. Es una forma creativa que no exige pasos previos, ni niveles de escolaridad. Es el lenguaje el que permite sintonizar lo diverso, el mapa es un texto. (García Barón, 2005, p. 5). 
Figura 2. Fotografía propia - Centro de apoyo Cayambe

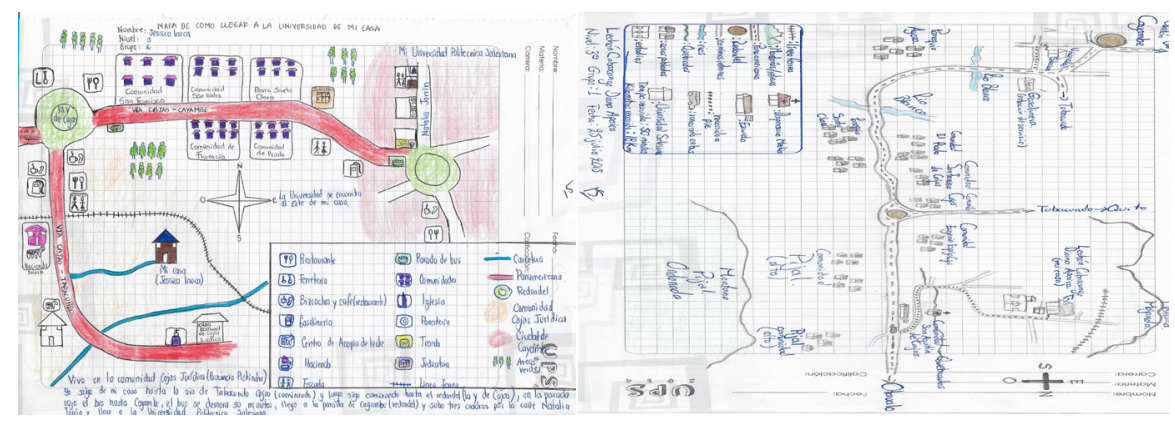

Fuente: Elaboración propia.

Por tanto, teniendo como base que el mapa también puede leerse como texto se convierte entonces en una estrategia didáctica que permite tanto el desarrollo de didácticas de enseñanza como de investigación en comunidad. El mapa se convierte en un proceso de construcción colectiva y llena de significados, a partir de los lugares referenciados, de los símbolos empleados.

En ese sentido la cartografía social: "Es una herramienta que permite vislumbrar cómo, en nuestros espacios, se están materializando las relaciones económicas, políticas, culturales, éticas, raciales y de género, resultantes de las transformaciones por las cuales pasa el mundo actual. También, analizar sus consecuencias. (...)" (Mancila y Habegger, 2006, p. 8).

Figura 3. Fotografía propia - Planos de recorrido

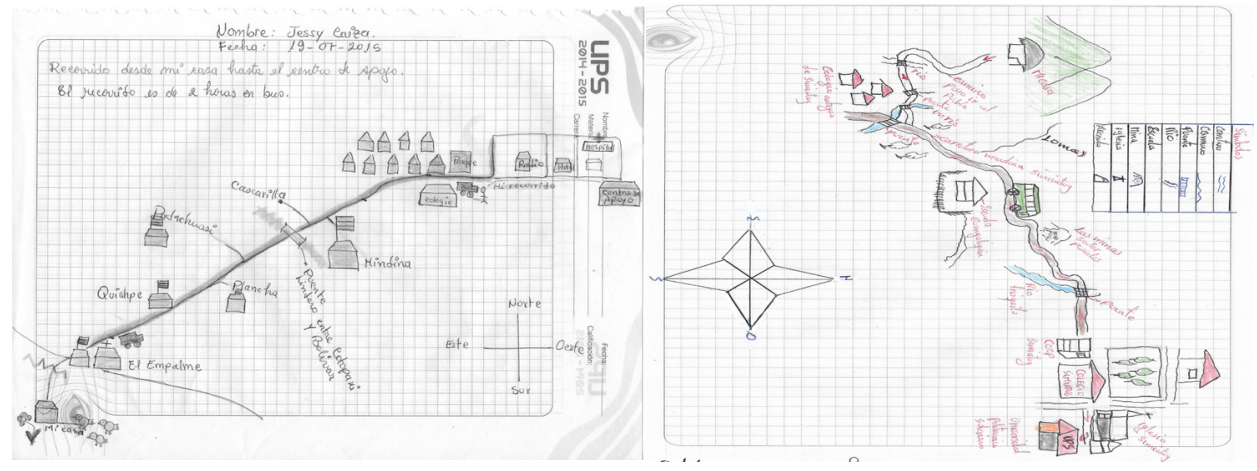

Fuente: Elaboración propia.

Se pretende que el uso de mapas, a partir de la cartografía social, incida en los espacios educativos generando rupturas con la enseñanza tradicional de la geografía en las escuelas y más aún en las escuelas interculturales bilingües. 
Teniendo en cuenta los cambios a nivel curricular y su aplicación en dichas instituciones, donde la enseñanza de las ciencias sociales y en particular de la geografía ha ido perdiendo relevancia frente a las ciencias exactas, y donde la geografía se convierte en una disciplina importante frente al reconocimiento como mencionamos anteriormente del territorio y de quienes lo integran. Lo que se evidencia en la organización comunitaria y las diversas actividades que los pobladores hacen en sus territorios, ya que se integran saberes tradicionales frente a la maquinaria y avance de la extracción de recursos por agentes externos a las comunidades.

Es importante entonces el reconocimiento como sujetos dentro de los territorios y que estos vean a la cartografía, como expone Boaventura de Sousa Santos, como una herramienta para la organización del territorio a partir de la integración de sus principales elementos, como lo son la escala, la proyección y la simbolización (De Sousa Santos, 1991). Lo que arroja ciertas bases para el uso de la cartografía social desde el trabajo pedagógico, permite orientar la construcción y uso de diversidad de mapas, desde los oficiales hasta los de construcción propia de los territorios:

Para que la geografía emerja como una forma de comprensión del mundo a partir de las dinámicas sociales en su dimensión espacial, es necesario adquirir habilidades que permitan realizar ejercicios analíticos que incluyan la espacialidad, de ello debe ocuparse la geografía escolar. (...) conceptos centrales para el desarrollo del raciocinio espacial los siguientes; lugar, paisaje, región, naturaleza, sociedad, territorio (Salgado Labra, 2011, p. 7).

Salgado (2011) destaca el concepto de "Lugar", en relación con la importancia frente a las percepciones y representaciones que los estudiantes tienen de su entorno, sus "espacios cercanos y cotidianos" para poder comprender situaciones específicas que se presentan en su contexto.

Figura 4. Fotografía propia - Centro de apoyo Simiatug

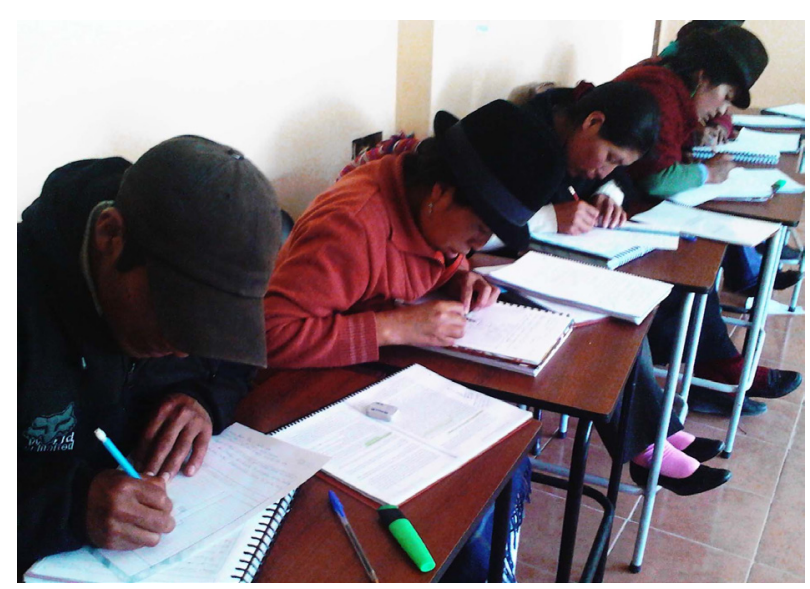

Fuente: Elaboración propia. 
Los anteriores conceptos y categorías son importantes para la reflexión y discusión de la cartografía social y su uso estrategia didáctica en la escuela, para la presente investigación que se reflejará en la práctica realizada y como aporte para los nuevos campos de saber geográfico llamados:

Campos Emergentes de la Geografía, los cuales promueven y lideran nuevas y diversas maneras en la comprensión del espacio y por tanto abogan por la instauración de la espacialidad como categoría central en geografía. Interesa en estas perspectivas la relación de los sujetos con su entorno, la vivencia y experiencia espacial, el espacio como producto socioeconómico y sociocultural, los lugares específicos en donde se desarrolla la vida de las personas (casa, calle, parque, barrio, plaza, etc.); a su vez exponen estas perspectivas geográficas un fuerte interés por el desarrollo de una educación geográfica que considere los escenarios escolares como constructores de la espacialidad en los sujetos, pero que a su vez sea capaz de desbordarlos de modo que los ciudadanos habitantes de los lugares cimienten una relación de diálogo con el espacio que ocupan, alcanzando en lo posible la condición de habitar los lugares. (Moreno Lache, Cely Rodríguez y Rodríguez Pizzinato, 2013, p. 144).

\section{CONCLUSIONES}

La investigación realizada permite evidenciar las diferentes habilidades de los estudiantes para expresarse, y desarrollar procesos propios de aprehendizaje de diversos conocimientos, por ejemplo, partiendo del uso de imágenes, gráficos y símbolos, entre otros, así como el proceso de formación de competencias de ubicación espacial y geográfica, así como el reconocimiento de los saberes propios y de sus territorios.

De esta manera la memoria colectiva de la comunidad perdura, a partir del reconocimiento de sus territorios. Los cuales cambian en muchas ocasiones por la incursión de personas fuera de la comunidad, generando nuevas dinámicas que afectan sus relaciones sociales y de relación con su entorno; que al reconocerlos pueden generar diversas situaciones y asimismo contribuir a la revitalización de sus territorios, a partir, del cuidado de los recursos que lo integran por parte de las poblaciones que los habitan.

La cartografía social se constituye en este sentido en una estrategia didáctica, como aporte a los nuevos campos de saber geográfico que aportan al reconocimiento de espacios y contextos en relación con la cotidianidad de quienes lo habitan. Tanto para su aplicación en espacios escolares, en la enseñanza de la geografía y las ciencias sociales en general, como para el caso de la investigación presentada, al permitir evidenciar las relaciones de la cotidianidad de los estudiantes y su roles como miembros de la comunidad, así como sus roles como estudiantes universitarios y de igual manera involucra a quienes comparten este camino, profesores, autoridades y compañeros, que aportan dentro del proceso de formación como docentes de las escuelas interculturales bilingües en sus territorios. 


\section{REFERENCIAS BIBLIOGRÁFICAS}

Barragán, D. \& Amador, J. (2014). La cartografía social-pedagógica: Una oportunidad para producir conocimiento y re-pensar la educación. Itinerario Educativo, (64), 127-141.

Carballeda, A. J. (2014). Cartografías e Intervención en lo social. En J. M. Diez Tetamanti, B. Escudero \& compiladores, Cartografía social. Investigación e intervención desde las Ciencias Sociales, métodos y experiencias de aplicación. Comodoro Rivadavia, Argentina: Universitaria de la Patagonia.

De Sousa Santos, B. (1991). Una cartografía simbólica de las representaciones sociales. Prolegómenos a una concepción posmoderna del derecho. Revista Nueva Sociedad, (116), 18-38.

Dussel, E. (2014). Cartografías del saber desde la transmodernidad. En B. Aguer (Ed.), Cartografías del poder y descolonialidad. Colección el desprendimiento. Buenos Aires, Argentina: Ediciones el Signo, 31-41.

Fals Borda, O. (2017). Campesinos de los Andes y otros escritos antológicos. Bogotá, Colombia: Universidad Nacional de Colombia. . (1998). Participación popular: retos del futuro. Compilación y análisis. Bogotá, Colombia: ICFES-IEPRI-Colciencias.

Freire, P. \& Faundez, A. (2013). Por una pedagogía de la pregunta. Crítica a una educación basada en respuestas a preguntas inexistentes. Buenos Aires, Argentina: Siglo Veintiuno editores.

García, C. (2005). La cartografía social en la práctica. Proyecto "Barrios del Mundo: Historias Urbanas". ENDA Colombia, Colombia.

Granda Merchán, S. \& Iza Remache, R. A. (2012). Los salesianos, la educación superior y los pueblos indígenas. En L. Vásquez, J. F. Regalado, B. Garzón, V. H. Torres \& J. Juncosa (Eds.), La presencia salesiana en el Ecuador. Perspectivas históricas y sociales. Cuenca, Ecuador: Abya-Yala.

Mancila, I. \& Habegger, S. (2006). El poder de la Cartografía Social en las prácticas contrahegemónicas o la Cartografía Social como estrategia para diagnosticar nuestro territorio.

Moreno Lache, N., Cely Rodríguez, A. \& Rodríguez Pizzinato, L. (2013). Pensar e indagar la construcción social del espacio: balances y retos. Revista Folios, (38), 141-156.

Salgado Labra, V. (2011). Construyendo prácticas pedagógicas críticas para la enseñanza de la geografía. Revista Geográfica de América Central, 1-12.

Santos, M. (2000). La naturaleza del espacio. Técnica y tiempo. Razón y emoción. Barcelona: Ariel S. A.

Tetamanti, J. M. (2014). Cartografía Social. Herramienta de intervención e investigación social compleja. El vertebramiento inercial como proceso mapeado. En J. M. Diez Tetamanti, B. y Escudero \& compiladores, Cartografía social. Investigación e intervención desde las Ciencias Sociales, métodos y experiencias de aplicación. (1a. edición ed.). Comodoro Rivadavia, Argentina: Universitaria de la Patagonia. 
